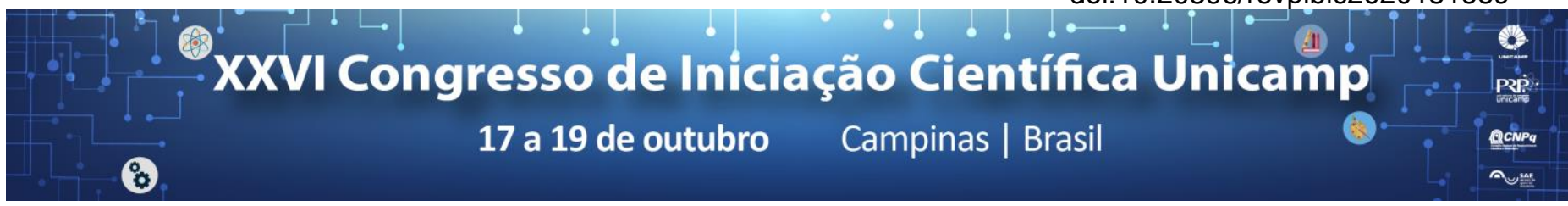

\title{
Thalamic Atrophy and Mood Changes in juvenile Systemic Erythematosus Lupus (SEL).
}

\section{Wolfgang Thyerre Pinto*, Aline Tamires, Danilo Rodrigo Pereira, Leticia Rittner, Dra Simone Appenzeller.}

\begin{abstract}
The thalamus is a neuroanatomic structure that performs many connections with the cerebral cortex and limbic structures. It is associated with sensorial, motor, emotional and motivational processes. In juvenile Systemic Erythematosus Lupus (SEL), the casualty and the diversity of neuropsychiatric symptoms are not fully established. The objective of this work is to evaluate the existence of structural changes in thalamus and its relation to depression, anxiety, and cognitive impairment in juvenile SEL. We concluded there is a reduction in thalamus volume in SLE and that reduction may contribute with the neuropsychiatric manifestations
\end{abstract}

Key words: Systemic Erythematosus Lupus, Anxiety, Central Nervous System.

\section{Introduction}

Systemic Lupus Erythematosus (SLE) is an autoimmune disease of inflammatory character due to a combination of environmental, genetic and hormonal factors [1-5], A wide spectrum of clinical manifestations with variable intensity can be observed $[2,5]$. The worst prognosis results from renal and central nervous system (CNS) impairment [6].

There are differences in the causality of neuropsychiatric symptoms and may be related to high and prolonged stress, CNS impairment and / or drug treatment $[7,8]$.

The objective of this work is to evaluate the existence of structural changes in thalamus and its relation to depression, anxiety, and cognitive impairment in childhood-onset Systemic Lupus Erythematosus (cSLE).

\section{Results and Discussion}

This is a cross-sectional study. A total of 72 patients were selected with diagnostic of cSLE. The control group was composed of 29 healthy people, age and sex matched. All patients and the control group were submitted to magnetic resonance imaging.

The thalamus was segmented using software "Freesurfer". Atrophy was defined when z-score $\leq-2$ standard deviations of the mean obtained from structures of the control individuals.

Mood disorders were evaluated through Beck depression and anxiety index. The cognitive impairment was evaluated using Montreal Cognitive Assessment. Medical records were reviewed for clinical, immunological and treatment related variables.

Statistical analyzes were performed according to the nature of the variables with $p \leq 0.05$ statistically significant.

The thalamic volume was significantly smaller in cSLE than in controls $(p \leq 0,001)$.

No association between thalamic volumes and autoantibodies in onset of disease was observed.

We observed atrophy in the thalamus in $1(3,4 \%)$ control sample, affecting the two symmetrical portions, and in $18(25 \%)$ patients, of whom 11 had symmetrical atrophy and 7 only in the right thalamus $(p=0,011)$.

Right thalamic atrophy was associated with anxiety $(p=0.047)$ and history of seizures $(p=0,022)$. A correlation between right thalamic volume and cognitive evaluation scores was observed $(p=0.007 ; r=0.364)$.

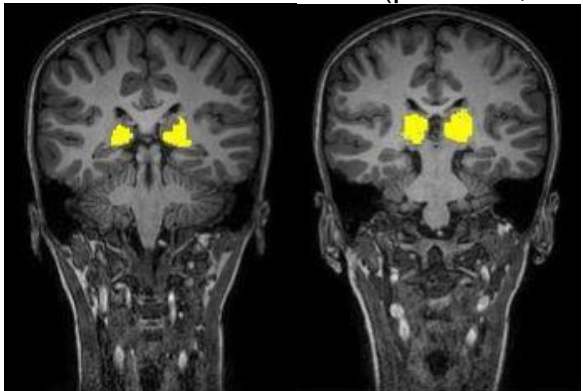

Image-1 patient group (“Freesurf"-segmentation)

\section{Conclusions}

We concluded that reduction in thalamus volume may contribute to the neuropsychiatric manifestations, requiring further investigations.

\section{Acknowledgement}

I thank God first for this opportunity and for helping me overcome the problems that have arisen. I am grateful for the support of Dr. Simone Appenzeller, PhD students Aline Tamires, Mariana Postal Zink de Souza and Cátia Nascimento, and Master student Jaqueline Amorim for help in the development of the project. This project is finance by FAPESP (Process:2017/04309-7; 02/01/2018 to 01/31/2019)

1. Benseler SM, Silverman ED. Systemic lupus erythematosus. Pediatr Clin North Am. 2005;52(2):443-67.

2. Borba EF, Latorre LC, Carlos J, Brenol T, Kayser C, Antonio N, et al Consenso de Lúpus Eritematoso Sistêmico Rev Bras Reum. 2008;55(1):196207.

3. Céspedes D, Ayache G, Pereira I. Alterações da Personalidade no Lúpus Eritematoso Sistêmico. 2005;55(67):313-8.

4. Munoz LE, Gaipl US, Franz S, Sheriff A, Voll RE, Kalden JR, et al. SLE A disease of clearance deficiency? Rheumatology. 2005;44(9):1101-7.

5. Sato EI, Bonfá ED, Tereza L, Costallat L, Antonio N, Carlos J, et al. Consenso brasileiro para o tratamento do lúpus eritematoso sistêmico ( LES ) 2002;42(6):362-70.

6. Appenzeller S, Costallat T. Análise de Sobrevida Global e Fatores de Risco para Óbito em 509 Pacientes com Lúpus Eritematoso Sistêmico ( LES ). Rev Bras Reumatol. 2004;44(3):198-205.

7. Lim LC, Lee T, Boey M: Psychiatric manisfestation of Systemic Lupus Erythematosus in Singapore:A cross - culture comparison. Br J Psychiatry 1991;159: 520-523.

8. HANLY J. G., McCURDY G, FOUGERE L., DOUGLAS J. THOMPSON K. Neuropsychiatric Events in Systemic Lupus Erythematosus: Attribution and Clinical Significance. The Journal of Rheumatology 2004;31(11): 2156-2162. 